This item was submitted to Loughborough's Research Repository by the author.

Items in Figshare are protected by copyright, with all rights reserved, unless otherwise indicated.

\title{
A method for forming distributed beams in time modulated planar arrays
}

PLEASE CITE THE PUBLISHED VERSION

https://doi.org/10.1109/TAP.2018.2871878

PUBLISHER

(c) IEEE

VERSION

AM (Accepted Manuscript)

LICENCE

CC BY-NC-ND 4.0

REPOSITORY RECORD

Clark, Ben, and James A. Flint. 2019. "A Method for Forming Distributed Beams in Time Modulated Planar Arrays". figshare. https://hdl.handle.net/2134/35087. 


\title{
A Method for Forming Distributed Beams in Time Modulated Planar Arrays
}

\author{
Ben Clark, James A. Flint, Senior Member, IEEE
}

\begin{abstract}
The sidebands which originate in time modulated arrays can be used for numerous applications, including multipath signal separation and directional power transfer. In the present paper, a method of simultaneously producing a series of beams that are steered towards unique two-dimensional directions is presented by developing the theory of time-modulated linear arrays to time-modulated planar arrays. A planar array is modelled as two linear arrays on orthogonal axes and the binomial and Dolph-Chebyshev time-weighting distributions are applied to a $5 \times 5$ element array. The output of the array is shown in both the time and frequency domains.
\end{abstract}

Index Terms-Array Signal Processing, Beamforming, Planar arrays, Time Modulation.

\section{INTRODUCTION}

The concept of Time Modulated Arrays (TMAs) was introduced by Shanks and Bickmore [1], [2], and is a method of beamforming by means of switching on and off elements in a conventional antenna array. This process transfers the power of an incoming or outgoing signal into a number of sidebands whose separation is governed by the switching frequency. In a receiver, the amount by which these bands are energised depends on the signal incident angle and consequently it is possible to determine this angle by analysis of the amplitude of each sideband.

TMAs have received increased research interest in recent years due to the switching methods being considerably less complex than phase-shifting and amplitude weighting methods. Research into TMAs has started to spread to multiple application domains such as signal processing [3] and communications systems [4]. Research has mainly focused on developing methods of controlling the array output.

Many different uses and techniques exist for TimeModulated Linear Arrays (TMLAs). Knowing the input response or output powers and steering angles of sidebands are of interest to applications such as directive power transfer [5], [6], Direction of Arrival (DoA) finding systems [7][11] and communications systems in multipath environments [12]. These applications make use of a TMLA's ability to simultaneously create multiple beams that are responsive in different angles relative to the array's broadside.

A time-modulated alternative to element amplitude weighting was demonstrated by Tong and Tennant [13]. It was shown that by adjusting the amount of time that individual elements

Manuscript received September 18, 2018. The authors are grateful for the financial support of Loughborough University's mini Centre for Doctorial Training (mCDT) in Digital Technologies.

Authors are with the School of Mechanical, Electrical and Manufacturing Engineering, Loughborough University, Leicestershire, LE11 3TU, UK; phone: +44(0)-150-922-7036; e-mail: BClark@ theiet.org. are turned on, the results correlated well to conventional array weighting achieved by signal amplification. This technique was demonstrated using well-known weighting distributions such as the Dolph-Chebyshev distribution [14] for a single axis.

It is possible to extend TMA concepts to Time Modulated Planar Arrays (TMPAs). TMPAs have similar benefits to TMLAs, however they have received relatively little research effort. To date, research has mainly focused on reducing the sidebands and sidelobes that occur during the switching process [15]-[17]. The present paper extends on existing knowledge by considering the case of multiple simultaneous beams steered in two broadside angles.

Poli et al. [18] showed that the calculations and design of TMPAs could be simplified by modelling the array as a multiplication of two TMLAs on orthogonal axes, where the signal's position is given in terms of spherical coordinate angles $\phi$ and $\theta$. Applying weighting distributions using time switching has also been applied in planar arrays [15], [19]. Since most of these works focus on reducing sidebands, elements are usually switched on simultaneously to receive a higher response at the centre frequencies.

The present paper develops the theory of a planar array as two linear axes and incorporates it into an array with an element-by-element switching pattern to produce a number of distributed beams. Each of these beams are steered towards a unique two-dimensional location and operate on different harmonic frequencies; therefore, the theory can be used in directional applications such as spatial filtering or DoA estimations. To make the planar array more easily comparable to its linear counterpart, the angular position of the signal is given in terms of broadside angles along orthogonal axes. The theory is extended further by incorporating well known linear array weighting systems into the planar array theory to show that the sidelobes of each of these beams can be controlled.

Section II provides a brief overview of the theory behind TMPAs. A switching order is provided for distributing multiple simultaneous beams and a set of equations for determining the steering angles of each harmonic in broadside angles and their equivalent in $\phi$ and $\theta$ angles is provided. The theory is concluded by demonstrating how these harmonic patterns can be controlled whilst keeping the same steered direction. Section III provides results obtained from a numerical simulation of a TMPA in both the time and the frequency domain. Finally, Section IV summarises the contribution of the paper. 


\section{THEORY}

\section{A. Background}

The planar uniform array factor of an $M \times N$ element array equally spaced with distances $d_{x}$ and $d_{y}$ on the $x$ and $y$ axes respectively can be derived from first principles and written as [15]:

$$
\begin{aligned}
A F(\theta, \phi, t)= & e^{j \omega_{0} t} \sum_{m=0}^{M-1} \sum_{n=0}^{N-1} u_{m n}(t) \\
& \times e^{j k \sin \theta\left(m d_{x} \cos \phi+n d_{y} \sin \phi\right)}
\end{aligned}
$$

where $\omega_{0}$ is the angular frequency of the primary signal, $k$ is its wave number, $\phi$ and $\theta$ are the signal azimuth and elevation angles respectively. $u_{m n}(t)$ is the switching function that turns each element either on or off in time which can be mathematically defined by:

$$
u_{m n}(t)= \begin{cases}1, & \tau_{m n, o n} \leq t<\tau_{m n, o f f} \\ 0, & \text { otherwise }\end{cases}
$$

where $\tau_{m n, o n}$ and $\tau_{m n, o f f}$ represent the switch on and off times for an element indexed by $(m, n)$. This function can be periodic with angular frequency $\omega_{s}=2 \pi / T_{s}$ where $T_{s}$ is the time taken to complete the switching sequence. This can be expressed as the sum of its Fourier coefficients with harmonic numbers indexed by $h$ :

$$
u_{m n}(t)=\sum_{h=-\infty}^{\infty} C_{m n} e^{j h \omega_{s} t}
$$

The coefficients $C_{m n}$ can be calculated as:

$$
C_{m n}=\frac{1}{T_{s}} \int_{0}^{T_{s}} u_{m n}(t) e^{-j h \omega_{s} t} d t
$$

To simplify the beam steering equations, this can also be modelled as the multiplication of two equations representing the array factors of orthogonal linear axes having switching functions $u_{m}(t)$ and $u_{n}(t)$ [18]. In the present paper, it is assumed that the array is illuminated in the far field from the two broadside angles $\beta_{x}$ and $\beta_{y}$ which are related to $\phi$ and $\theta$ angles as shown in Fig. 1 by:

$$
\begin{aligned}
& \beta_{x}=\sin ^{-1}(\sin \theta \cos \phi) \\
& \beta_{y}=\sin ^{-1}(\sin \theta \sin \phi)
\end{aligned}
$$

It can be shown that (1) can be expressed as:

$$
\begin{aligned}
A F\left(\beta_{x}, \beta_{y}, t\right)= & e^{j \omega_{0} t} \sum_{m=0}^{M-1} \sum_{n=0}^{N-1} u_{m n}(t) \\
& \times e^{j k\left(m d_{x} \sin \beta_{x}+n d_{y} \sin \beta_{y}\right)}
\end{aligned}
$$

Observing that $u_{m n}=u_{m} \cdot u_{n}$, it can be further shown that

$$
\begin{aligned}
A F\left(\beta_{x}, \beta_{y}, t\right)= & e^{j \omega_{0} t} \sum_{m=0}^{M-1} u_{m}(t) e^{j k m d_{x} \sin \left(\beta_{x}\right)} \\
& \times \sum_{n=0}^{N-1} u_{n}(t) e^{j k n d_{y} \sin \left(\beta_{y}\right)}
\end{aligned}
$$

which will form the basis of predicting the steering angle of each harmonic produced by the TMPA. The following sections

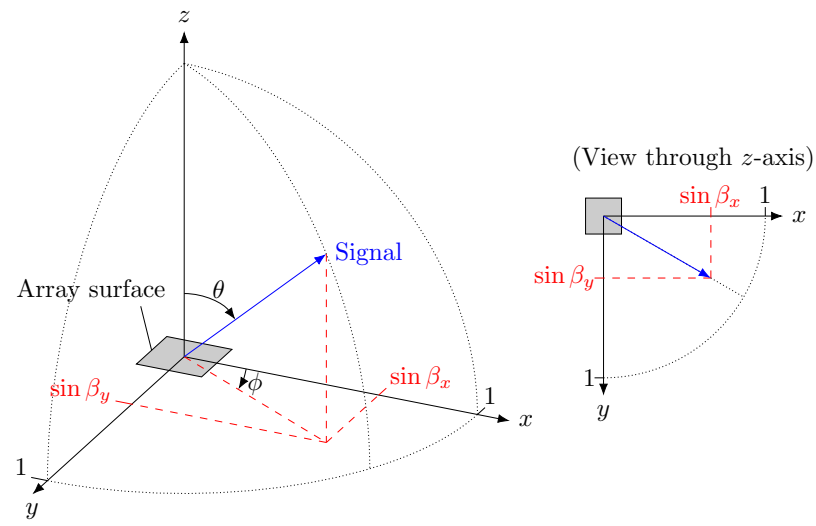

Fig. 1. Coordinate system showing the relationship between $x$ or $y$-axis broadside angles $\left(\beta_{x}\right.$ and $\left.\beta_{y}\right)$ and spherical coordinate angles $(\phi$ and $\theta)$.

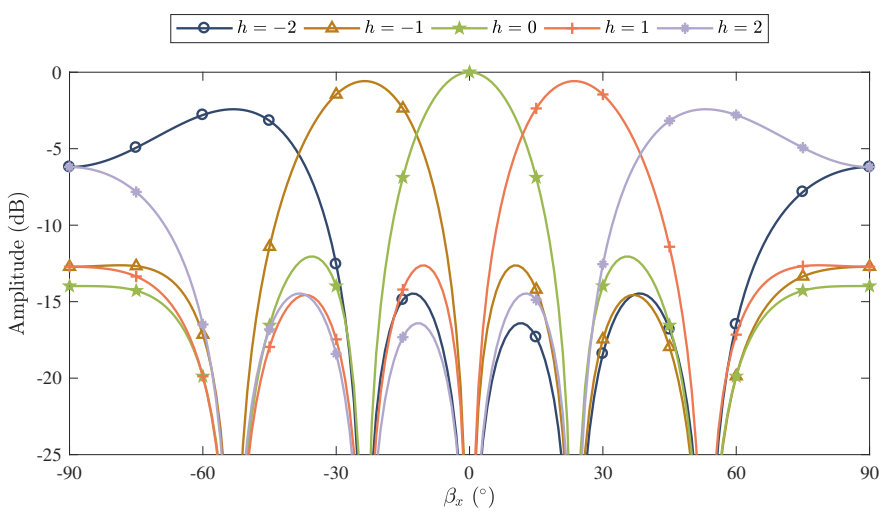

Fig. 2. The array factor of the sidebands generated by a 5 element TMLA when switched uniformly element-by-element down a single axis.

make use of this form to generate unique beam patterns across the range of harmonics.

\section{B. Generating Beams in Unique Directions}

As described by Tong and Tennant [13], the sequential switching of a linear array with periodic on times causes the beams of sidebands to form in unique directions across a single broadside axis. As shown in [9], the linear array switched in this way with $M$ elements laying on the $x$-axis and having equal element weightings will generate several sidebands with an array factor:

$$
\begin{aligned}
A F\left(\beta_{x}, t\right)= & \frac{1}{M} \sum_{h=-\infty}^{\infty} e^{j\left(\omega_{0}+h \omega_{s}\right) t} \operatorname{sinc}\left(\frac{\pi h}{M}\right) \\
& \times \sum_{m=0}^{M-1} e^{2 \pi j m r_{x}\left[\sin \left(\beta_{x}\right)-\frac{h}{M r_{x}}\right]}
\end{aligned}
$$

where sinc $\chi$ is the non-normalised cardinal sine function and $r_{x}$ is the ratio of element spacing $d_{x}$ to the wavelength $\lambda$.

Fig. 2 shows the array factor for each sideband in a five element TMLA. These sideband beams each have a main lobe that points to a unique direction relative to the broadside. A second, independent TMLA can be used along the $y$-axis in order to obtain two-dimensional data. If it is desirable to 


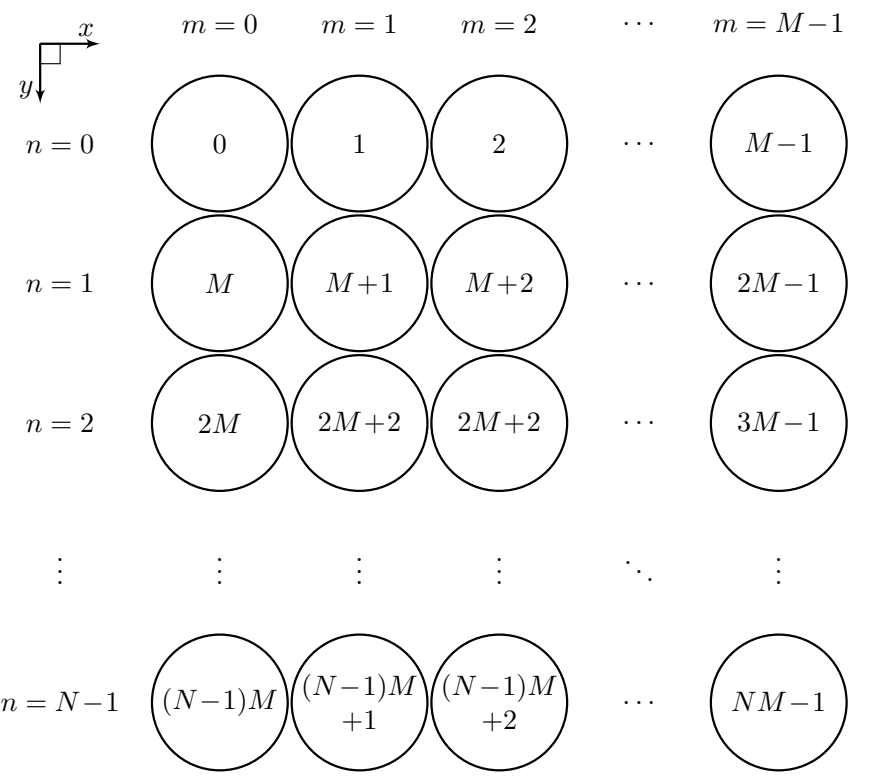

Fig. 3. The proposed switching order for an $M \times N$ element array starting at index 0 .

use these beams simultaneously (e.g. for applications such as DoA estimation), then the switching of the respective element in each axis must also occur simultaneously and the output of each array using this technique needs to be acquired and analysed separately.

In the present paper, it is proposed that each of the elements in a planar array are switched on then off in sequence, elementby-element in the order $i=0,1,2, .$. , first by switching left to right on the top row of elements, then continuing to the next row until all elements have been switched once. This sequence is shown in Fig. 3.

If the switching pattern is to be periodic, the definition of $C_{m n}$ can be obtained using (2) and (4). Since, $C_{m n}$ is independent of the element position, its definition for a two-dimensional array with specific on times is the same as a linear array having the same number of elements and equivalent on times. Since the element index can be calculated as $i=(M n)+m$ then $C_{m n}$ can be calculated as:

$$
C_{m n}(t)=\operatorname{sinc}\left(\frac{\pi h}{M N}\right) e^{-\frac{j 2 \pi h[(M n)+m]}{M N}}
$$

The normalised array factor for a planar array switched in this manner is obtained by combining (3), (7) to obtain the switching function in terms of the sum of its Fourier components:

$$
u_{m n}(t)=\sum_{h=-\infty}^{\infty} \operatorname{sinc}\left(\frac{\pi h}{M N}\right) e^{-\frac{2 j \pi h[(M n)+m]}{M N}} e^{j h \omega_{s} t}
$$

and then substituting this expression for $u_{m n}(t)$ into (10) to produce:

$$
\begin{aligned}
A F\left(\beta_{x}, \beta_{y}, t\right)= & \frac{1}{M N} \sum_{h=-\infty}^{\infty} e^{j\left(\omega_{0}+h \omega_{s}\right) t} \operatorname{sinc}\left(\frac{\pi h}{M N}\right) \\
& \times \sum_{m=0}^{M-1} \sum_{n=0}^{N-1} e^{-\frac{2 \pi j h[(M n)+m]}{N M}} \\
& \times e^{j k\left(m d_{x} \sin \beta_{x}+n d_{y} \sin \beta_{y}\right)}
\end{aligned}
$$

In an array of $M \times N$ elements, it is expected that there will be $M \times N$ different sidebands numbered in the range of $\pm \frac{M \times N}{2}$. The switching of elements along the $x$-axis is $M$ times faster than the switching of elements along the $y$-axis. As a consequence, changes in signal position along the $x$ axis broadside will cause changes in power across a larger frequency range than if the signal position were to change across the $y$-axis broadside. When modelling these harmonics to represent different axes $h_{x}$ will be in the range of $\pm \frac{M}{2}$ and $h_{y}$ will be in the range of $\pm \frac{N}{2}$. Assuming that $h_{x}$ and $h_{y}$ are both integers within the ranges described, their relationship with the actual sideband number $h$ can be written as:

$$
h=N h_{x}+h_{y}
$$

For example in a $5 \times 5$ element array, measuring the power in the sideband $h=2$ is the equivalent to measuring the separated sidebands $h_{x}=0$ and $h_{y}=2$, however measuring at $h=3$ is equivalent of measuring the separated sidebands $h_{x}=1$ and $h_{y}=-2$. A normalised array factor using these separated sidebands can be obtained and simplified to:

$$
\begin{aligned}
A F\left(\beta_{x}, \beta_{y}, t\right)= & \frac{1}{M N} \sum_{h=-\infty}^{\infty} e^{j\left(\omega_{0}+h \omega_{s}\right) t} \operatorname{sinc}\left(\frac{\pi h}{N M}\right) \\
& \times \sum_{m=0}^{M-1} e^{2 \pi j m r_{x}\left[\sin \left(\beta_{x}\right)-\frac{h_{x}+h_{y} / N}{M r_{x}}\right]} \\
& \times \sum_{n=0}^{N-1} e^{2 \pi j n r_{y}\left[\sin \left(\beta_{y}\right)-\frac{h_{y}}{N r_{y}}\right]}
\end{aligned}
$$

where $r_{y}$ is the ratio of the element spacing on the $y$-axis $d_{y}$ to the wavelength $\lambda$ of the signal.

Fig. 4 shows the main beam steering angles for each of the sidebands originating from a $5 \times 5$ element array. It is important to note that when using the above equations, $\left|\beta_{x}\right|+\left|\beta_{y}\right| \leq 90^{\circ}$ (the boundary of which is shown as a dotted line) and these coordinates map to a single point on a hemisphere. The circled points on the figure that lie beyond the dotted line indicate beams that do not have a mappable point.

It can be seen that each sideband has a unique two dimensional steering angle, but sets of beams may share the same $y$-axis broadside angle. This is contrary to using the beams of two independent TMLAs, where it is expected that the $\beta_{x}$ and $\beta_{y}$ combinations would form a square grid. Switching along two different axes using the sequence described has the effect of skewing the positions of the faster switching axis (i.e. the $x$-axis). Fig. 5 shows the differences in beam position and amplitude along the $x$-axis when looking at different $h$ values.

Whilst these beams are different in amplitude and position, they share some of the same traits as would be found in a pair 


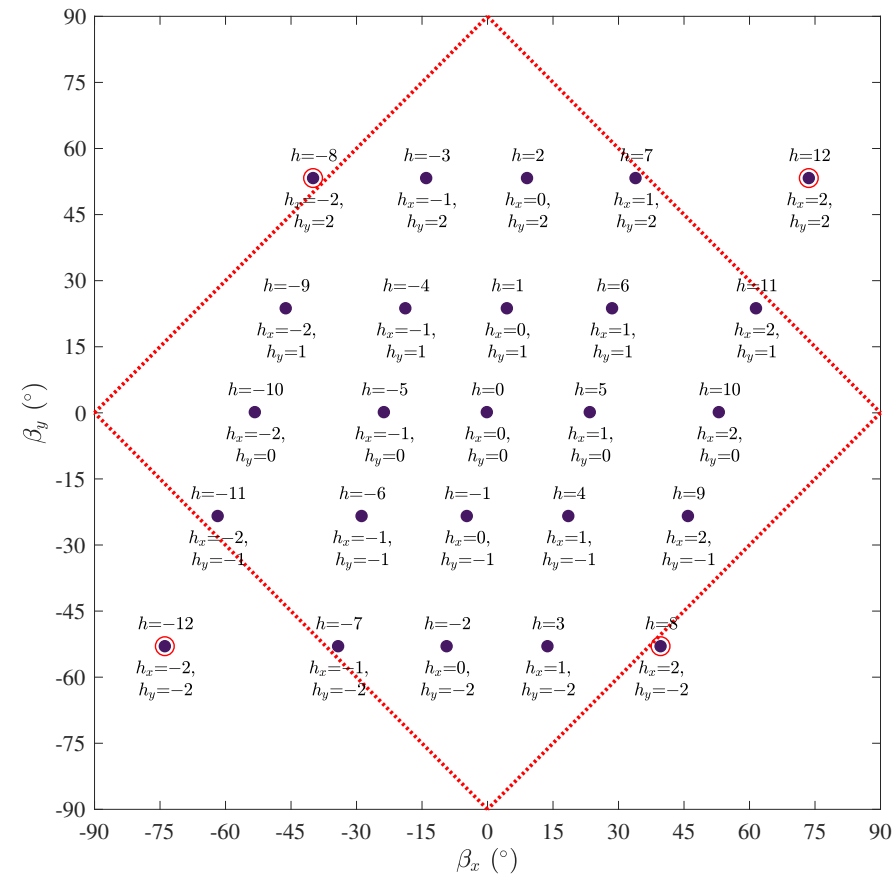

Fig. 4. Harmonic beam steering angles of a $5 \times 5$ element array under the proposed switching scheme.

of orthogonal TMLAs. At the locations where each sideband is maximised, all other sidebands are minimised. From (14) it can be shown that for each sideband, these locations map to unique combinations of $\beta_{x}$ and $\beta_{y}$ and these will occur at:

$$
\begin{gathered}
\beta_{y, \max }\left(h_{y}\right)=\sin ^{-1}\left(\frac{h_{y}}{r_{y} N}\right) \\
\beta_{x, \max }\left(h_{x}, h_{y}\right)=\sin ^{-1}\left(\frac{h_{x}+h_{y} / N}{r_{x} M}\right)=\sin ^{-1}\left(\frac{h}{r_{x} M N}\right)
\end{gathered}
$$

The maximum amplitude of these beams normalised to the main beam at the centre harmonic is:

$$
\max \left(A F_{h}\right)=\operatorname{sinc}\left[\frac{\pi\left(N h_{x}+h_{y}\right)}{M N}\right]=\operatorname{sinc}\left(\frac{\pi h}{M N}\right)
$$

Since the relative amplitude of each beam is known, then each harmonic can be normalised during a post-processing stage to have the same amplitude response across each sideband.

\section{Simultaneous Control of Sidelobes on Each Sideband}

As shown by Tong and Tennant [13], so long as the on times for each element are distributed evenly across the switching sequence, the weighting for each element can be adjusted by decreasing the total amount of time that it is switched on. Each element is on for a fraction of its allocated time depending on the weighting needed.

In an example, if three elements with equally distributed on times required the weighting $\{1,3,1\}$, the switching period would take place over nine quantised periods of time as shown in Fig. 6. The first element would switch on for one period, switch off and then wait two periods before switching to the next element, where it is on for three more periods. After this, it is switched off and the third element is switched on at the same time for a single period. The pattern can then be repeated after waiting an additional two periods. This switching pattern can be in practice controlled by using RF switches.

In conventional planar arrays, the weighting of well known distributions such as the binomial or Dolph-Chebyshev distributions can be calculated as a multiplication of two linear array weighting distributions [20].

Just as in linear arrays, weighting distributions for TMPAs may be realised by changing the amount of time that each element is on for [18], [21]. If a function $W(i)$ stipulates the amount of on time needed for element $i$ in the sequence of a TMLA, then the weighting for each element in a planar array with the switch sequence described in Section II-B is simply:

$$
W(M n+m)=W(m) \times W(n)
$$

Fig. 7 shows the weighting needed to achieve either a binomial, where the sidelobes are eliminated, or a DolphChebyshev array, where the sidelobes are set to a specific level relative to the main beam. In this paper, it is proposed that one element is on at any given time, and therefore weighting for planar array using switching is similar to the linear example shown in Fig. 6, but where the elements are switched in the order shown in Fig. 3.

The binomial distribution for each element $i$ on a single axis with $I$ elements can be calculated as:

$$
W(i)=\frac{I-1 !}{[(I-1)-i] ! i !}
$$

In the present paper, Bresler's method [22] has been used to calculate the individual Dolph-Chebyshev distribution weights.

\section{NumericAl RESUlts}

In this section a $5 \times 5$ element array has been simulated using discrete-time sample generation and results including array weighting techniques are discussed.

A source producing a single sinusoidal frequency of $1 \mathrm{GHz}$ was used, impinging on a $5 \times 5$ isotropic element array with inter-element spacing set at $\lambda / 2$. Each element was sampled for a specific amount of time before switching to the next element in the sequence as shown in Fig. 3. The switching of elements occurred at $250 \mathrm{MHz}$, creating a harmonic frequency of $10 \mathrm{MHz}$ and the power in each of these sidebands were measured using DFTs. Although the switching on times of elements remained fixed, three patterns were tested, where the amount of time that each element is sampled varies depending on the following weighting distributions:

1) Flat Array - Each element is sampled for the full amount of time it is switched on, sampling of the next element in the sequence occurs immediately after.

2) Binomial Array - Each element is sampled for a fraction of the allocated time proportional to the values indicated in Fig. 7(a).

3) Dolph-Chebyshev Array - Each element is sampled for a fraction of the allocated time proportional to the values indicated in Fig. 7(b). 


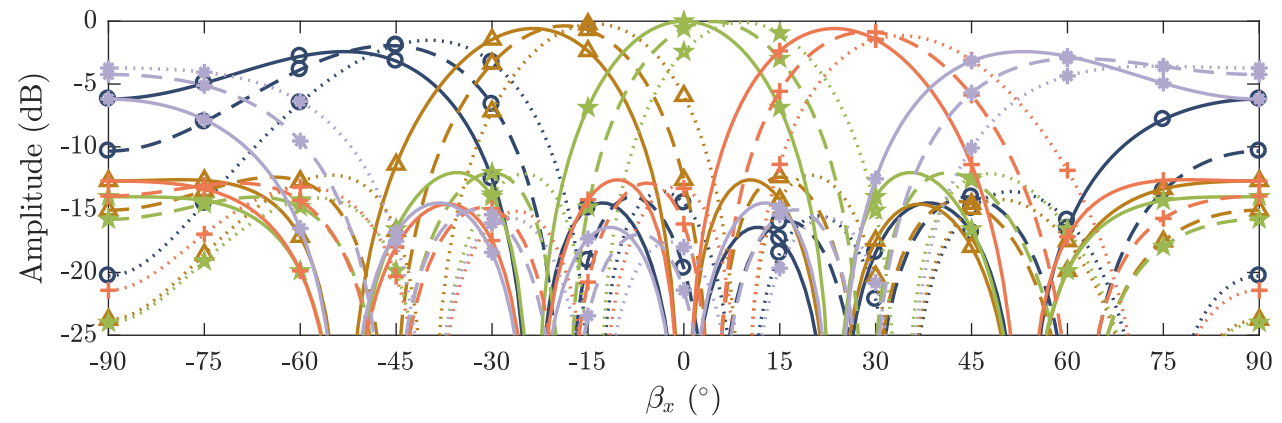

$$
\begin{aligned}
& \text { - } h=-10\left(h_{y}=0, h_{x}=-2\right) \\
& -\Theta-h=-9\left(h_{y}=1, h_{x}=-2\right) \\
& \cdots \bullet \cdots h=-8\left(h_{y}=2, h_{x}=-2\right) \\
& \triangle \quad h=-5\left(h_{y}=0, h_{x}=-1\right) \\
& -\Delta-h=-4\left(h_{y}=1, h_{x}=-1\right) \\
& \cdots \Delta \cdots h=-3\left(h_{y}=2, h_{x}=-1\right) \\
& \text { ¿ } h=0\left(h_{y}=0, h_{x}=0\right) \\
& -\star-h=1\left(h_{y}=1, h_{x}=0\right) \\
& \text {...... } h=2\left(h_{y}=2, h_{x}=0\right)
\end{aligned}
$$

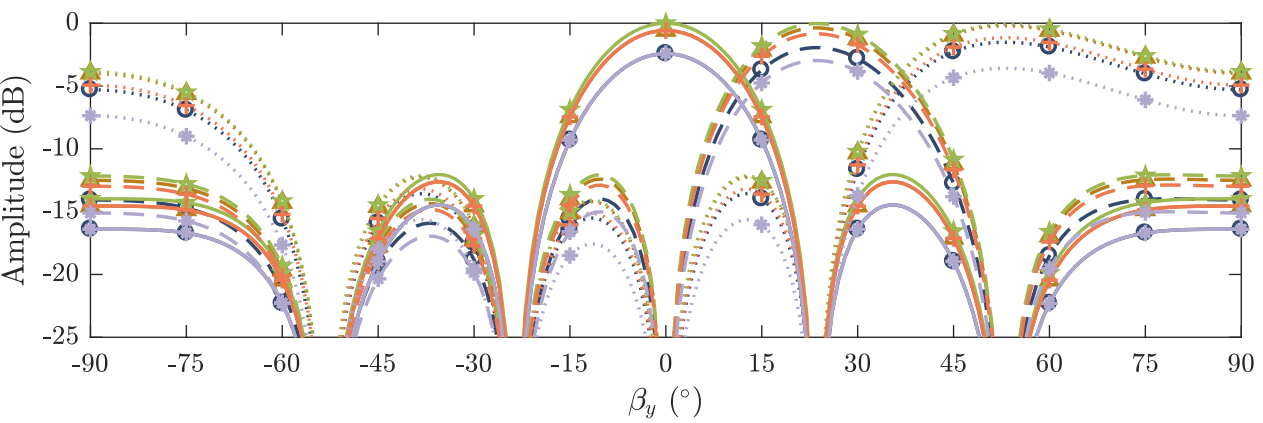

Fig. 5. The maximum possible response of selected sidebands when analysing different broadside axes. Negative $h_{y}$ values are not shown for clarity.

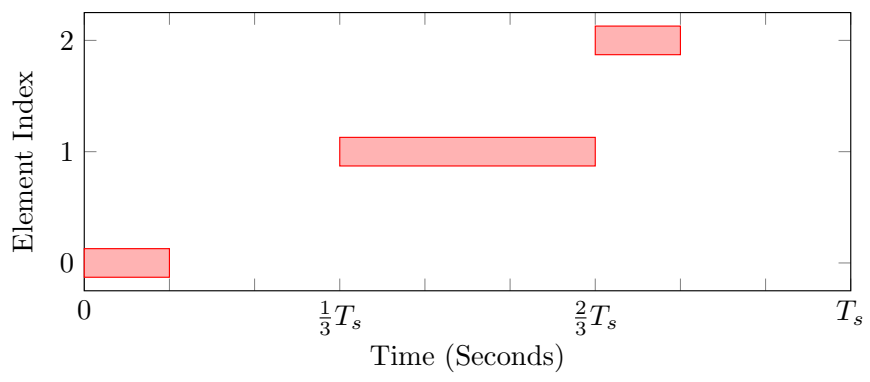

Fig. 6. Switching times of a three element array with a weighting of $\{1,3,1\}$. A bar indicates the time that a particular element is switched on.

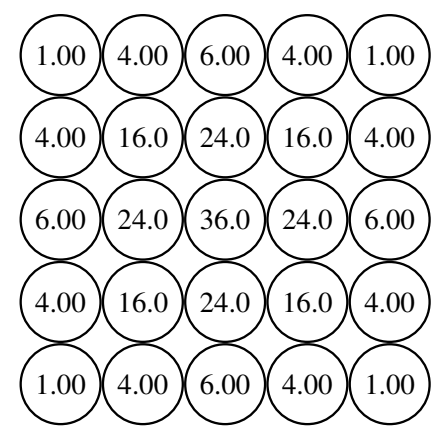

(a)

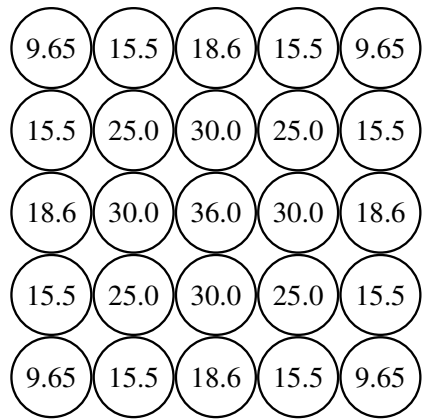

(b)
Fig. 7. Weighting distributions needed to achieve a $5 \times 5$ element (a) binomial and (b) $-20 \mathrm{~dB}$ Dolph-Chebyshev array.

\section{A. Time Domain}

Fig. 8 shows the array output in the time domain when the signal is directed towards the array at an angle of $\beta_{x}=$ $30^{\circ}, \beta_{y}=30^{\circ}$ (this corresponds to $\phi=\theta=45^{\circ}$ ). In the binomial and Dolph-Chebyshev distributions it is straightforward

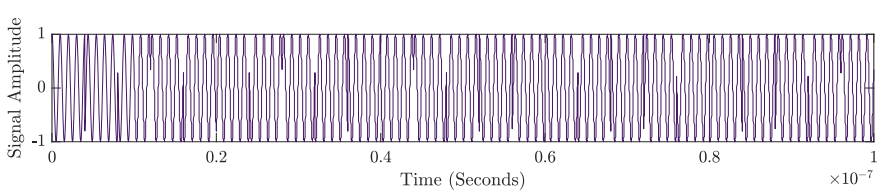

(a)

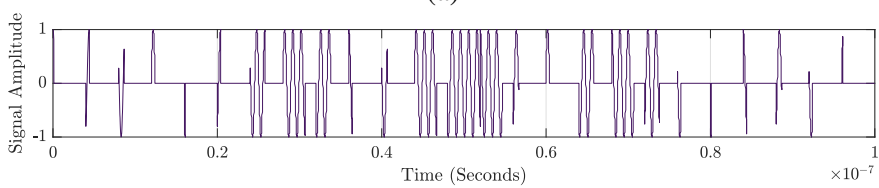

(b)

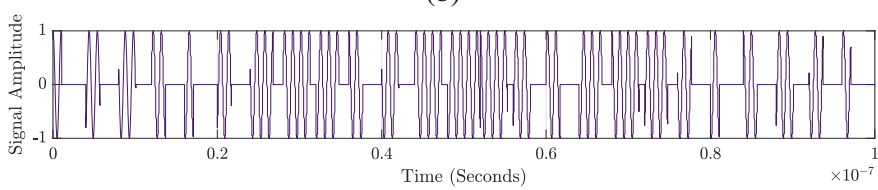

(c)

Fig. 8. Time domain output of a $5 \times 5$ time-modulated array with different weighting distributions: (a) flat, (b) binomial, (c) Dolph-Chebyshev.

to observe where switching occurs.

It can also be seen that in the Binomial distribution case, the array spends more time switched off than on. The ratio of on time to off time is $28.4 \%$, which yields a relatively low amount of received power. This ratio is significantly better in the Dolph-Chebyshev distributed array, at 54.8\%.

\section{B. Frequency Domain}

A set of 25 DFT Amplitudes were collected in the range of $0.88-1.12 \mathrm{GHz}$ (corresponding to each combination of $x$ and $y$ axis harmonics) for each angular position of the source. Fig. 9 shows the array response after performing a DFT at two different harmonics. 


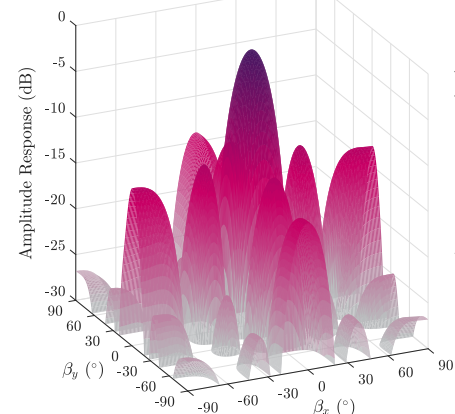

(a)

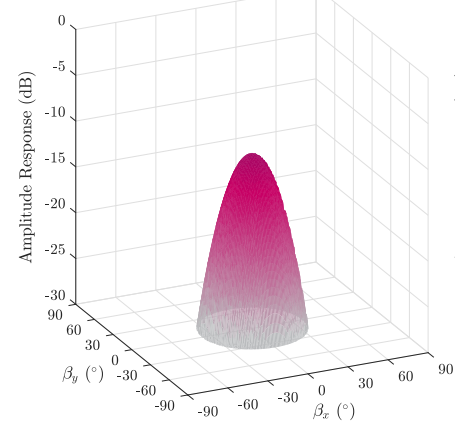

(c)

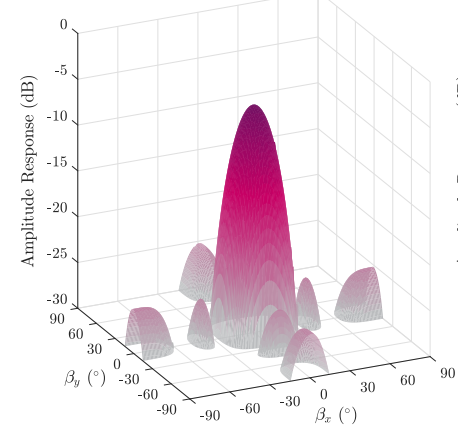

(e)

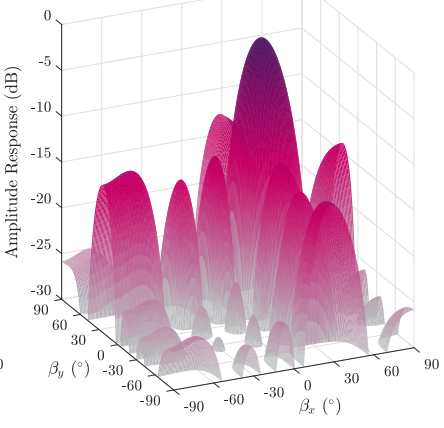

(b)

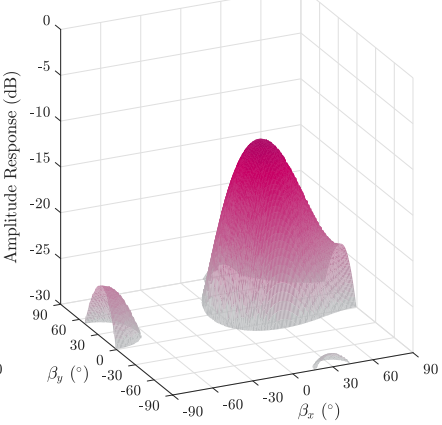

(d)

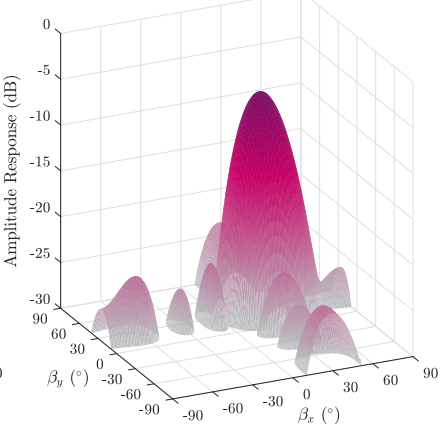

(f)
Fig. 9. Beam patterns of a $5 \times 5$ element array with three different timeweighting distributions and two different scanning frequencies: (a) Flat ( $h=$ 0 ), (b) Flat $(h=6)$, (c) Binomial $(h=0)$, (d) Binomial $(h=6)$, (e) Dolph-Chebyshev $(h=0)$, (f) Dolph-Chebyshev $(h=6)$.

At $h_{x}=0, h_{y}=0(h=0)$, it is clear that the array is most responsive at $\beta_{x}=0^{\circ}, \beta_{y}=0^{\circ}$, whereas at $h_{x}=1, h_{y}=1$ $(h=6)$, the point where the array is most responsive is at $\beta_{x}=28.69^{\circ}, \beta_{y}=23.58^{\circ}$. The effects of the time weighting are also in good agreement with the theory; in the binomial distribution, the sidelobes have been completely eliminated in every harmonic sideband, at the expense of producing a larger beam-width. The Dolph-Chebyshev distribution has a reduced level of side-lobes, and has a beam-width which is only slightly larger than the flat distribution.

Table I shows the amplitudes relative to the maximum possible power received by the array (i.e. at $h_{x}=h_{y}=\beta_{x}=$ $\left.\beta_{y}=0\right)$ and the half-power broadside beam-widths. Since the maximum amplitude at $h=0$ should be equal to the ratio of elements being on to elements being off (as mentioned in Section III-A), the binomial distribution significantly reduces the total power at the array. The slight discrepancies between

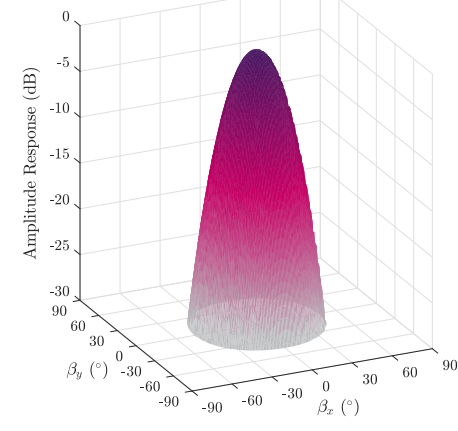

(a)

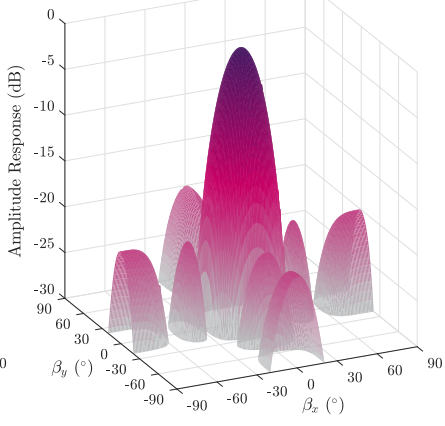

(b)
Fig. 10. Normalised Beam patterns of a $5 \times 5$ element array at $h=0$ for two different time-weighting distributions. (a) Binomial, (b), Dolph-Chebyshev.

TABLE I

AMPLITUDES OF EACH PATTERN IN DECIBELS RELATIVE TO FLAT DISTRIBUTION, AND THE HALF-POWER BEAM WIDTHS IN DEGREES.

\begin{tabular}{ccc}
\hline Weighting Distrbution & $\begin{array}{c}\text { Maximum Amplitude } \\
h=0\end{array}$ & $\begin{array}{c}\text { dB beam-width }) \\
h=6\end{array}$ \\
\hline Flat & $0.00\left(21^{\circ}\right)$ & $-0.84\left(23^{\circ}\right)$ \\
Binomial & $-10.92\left(30^{\circ}\right)$ & $-11.41\left(35^{\circ}\right)$ \\
Dolph-Chebyshev $(-20 \mathrm{~dB})$ & $-5.19\left(24^{\circ}\right)$ & $-5.73\left(27^{\circ}\right)$ \\
\hline
\end{tabular}

the maximum amplitude of the beams at $h=0$ and the ratios calculated in Section III-A are mainly due to the effects of sampling in the simulation. Fig. 10 shows the response of the central frequency when the incoming signal is amplified to counteract this ratio (i.e. by $5.19 \mathrm{~dB}$ for Dolph-Chebyshev, and $10.92 \mathrm{~dB}$ for binomial), and highlights the useful properties of each distribution when compared to the flat distribution.

From Table II it can be seen that the target sidelobe level specified for the Dolph-Chebyshev array is only achieved at the centre harmonic, the levels of each sidelobe have still been greatly reduced, and only vary by small quantities. Where only one unique beam per harmonic is desired, the Dolph-Chebyshev distribution makes a good alternative to the binomial distribution since the power received in the main beam direction is significantly greater compared to the power at its sidelobes or at the main beam of the binomial array in the same sideband.

\section{CONCLUSIONS}

A method of producing predictable simultaneous beam patterns has been proposed and results of numerical simulations have been given which show good agreement with the theory. The results and the theory have assumed that the switching

TABLE II

SIDELOBE LEVELS OF EACH PATTERN IN DECIBELS. VALUES IN BRACKETS INDICATE LEVELS RELATIVE TO THE PATTERN'S MAIN BEAM.

\begin{tabular}{ccc}
\hline Weighting Distribution & \multicolumn{2}{c}{ Sidelobe Amplitude } \\
& $h=0$ & $h=6$ \\
\hline Flat & $-11.54(-11.54)$ & $-12.56(-11.72)$ \\
Binomial & - & - \\
Dolph-Chebyshev (-20 dB) & $-25.22(-20.03)$ & $-23.54(-17.81)$ \\
\hline
\end{tabular}


between elements is ideal (e.g. instantaneous with no losses). The realisation of non-ideal arrays requires careful design [23].

It has been demonstrated that switching a planar array element-by-element is a simple way of obtaining unique beams for each harmonic. Although a broadband signal is scattered into different harmonics with different steering positions, these can be predicted using (15) and (16), where each frequency of the component signal will have a different value for $r_{x}$ and $r_{y}$.

Applying a binomial weighting to this switching pattern gives a solution for when no sidelobes are desired. Due to the ratios of on times between the corner and central elements, much of the signal capturing time is spent receiving no power at all, and thus the array captures a relatively small amount of power. The Dolph-Chebyshev distribution offers a compromise between obtaining low sidelobes and maximising power in the main beam, just as it does with conventional arrays but for each sideband.

It may also be beneficial to mirror the switching pattern or rotate the switch axis, (i.e. starting from the bottom of the array instead of the top) on the same array and route the signals to a secondary output. Not only will this increase the time efficiency of the array as described by [24] but the collection of harmonic beams in this secondary output would be mirrored compared to the first, allowing a processor to compare between closely situated beams, or to tune into a signal's position more precisely.

\section{REFERENCES}

[1] H. E. Shanks and R. W. Bickmore, "Four-Dimensional Electromagnetic Radiators," Can. J. Phys., vol. 37, no. 3, pp. 263-275, March 1959.

[2] H. E. Shanks, "A new technique for electronic scanning," IRE Trans. Antennas Propag., vol. 9, no. 2, pp. 162-166, March 1961.

[3] W.-Q. Wang, H. C. So, and A. Farina, "An Overview on Time/Frequency Modulated Array Processing," IEEE J. Sel. Top. Signal Process., vol. 11, no. 2, pp. 228-246, March 2017.

[4] R. Maneiro-Catoira, J. Brégains, J. A. García-Naya, and L. Castedo, "Time Modulated Arrays: From their Origin to Their Utilization in Wireless Communication Systems," Sensors, vol. 17, no. 3, p. 590, March 2017.

[5] R. Marchukov, D. Masotti, and A. Costanzo, "Dynamic wireless power transfer by time-modulated arrays," in 2015 IEEE Int. Symp. Antennas Propag. Usn. Natl. Radio Sci. Meet. IEEE, July 2015, pp. 808-809.

[6] D. Masotti, A. Costanzo, M. Del Prete, and V. Rizzoli, "TimeModulation of Linear Arrays for Real-Time Reconfigurable Wireless Power Transmission," IEEE Trans. Microw. Theory Tech., vol. 64, no. 2, pp. 1-12, 2016.

[7] G. Li, S. Yang, and Z. Nie, "Direction of Arrival Estimation in Time Modulated Linear Arrays With Unidirectional Phase Center Motion," IEEE Trans. Antennas Propag., vol. 58, no. 4, pp. 1105-1111, April 2010.

[8] C. He, X. Liang, Z. Li, J. Geng, and R. Jin, "Direction Finding by Time-Modulated Array With Harmonic Characteristic Analysis," IEEE Antennas Wirel. Propag. Lett., vol. 14, pp. 642-645, 2015.

[9] B. Clark and J. A. Flint, "Acoustical Direction Finding with TimeModulated Arrays," Sensors, vol. 16, no. 12, p. 2107, 2016.

[10] D. Ni, S. Yang, Y. Chen, and F. Yang, "Direction finding based on TMAs with reconfigurable angle-searching range and bearing accuracy," Electron. Lett., vol. 53, no. 3, pp. 130-132, February 2017.

[11] C. He, X. Liang, W. Zhu, G. Geng, R. Jin, A. Cao, and H. Di, "Direction finding by time modulated linear array," in 2017 IEEE International Symposium on Antennas and Propagation USNC/URSI National Radio Science Meeting, July 2017, pp. 315-316.

[12] R. Maneiro-Catoira, J. C. Bregains, J. A. García-Naya, L. Castedo, P. Rocca, and L. Poli, "Performance Analysis of Time-Modulated Arrays for the Angle Diversity Reception of Digital Linear Modulated Signals," IEEE J. Sel. Top. Signal Process., vol. 11, no. 2, pp. 247-258, 2017.
[13] Y. Tong and A. Tennant, "Simultaneous control of sidelobe level and harmonic beam steering in time-modulated linear arrays," Electron. Lett. vol. 46, no. 3, p. 200, 2010.

[14] C. Dolph, "A Current Distribution for Broadside Arrays Which Optimizes the Relationship between Beam Width and Side-Lobe Level," Proc. IRE, vol. 34, no. 6, pp. 335-348, June 1946.

[15] L. Poli, P. Rocca, L. Manica, and A. Massa, "Handling Sideband Radiations in Time-Modulated Arrays Through Particle Swarm Optimization," IEEE Trans. Antennas Propag., vol. 58, no. 4, pp. 1408-1411, April 2010.

[16] E. Afacan, "Sidelobe Level and Sideband Optimization for Thinned Planar Antenna Arrays Using Time Modulation," 2013 7th Eur. Conf. Antennas Propag., no. Eucap, pp. 328-330, 2013.

[17] D. Ni, S. Yang, J. Li, J. Guo, and Z. Nie, "An efficient approach for synthesizing irregularly shaped patterns based on 4D arrays," in 2015 IEEE Int. Conf. Commun. Probl. IEEE, October 2015, pp. 133-136.

[18] L. Poli, P. Rocca, L. Manica, and A. Massa, "Time modulated planar arrays Analysis and optimisation of the sideband radiations," IET Microwaves, Antennas Propag., vol. 4, no. 9, p. 1165, 2010.

[19] Q. Zhu, S. Yang, M. Huang, and Z. Nie, "Synthesis of Low Sidelobe Time Modulated Planar Arrays with Uniform Amplitude and Subsectional Optimized Time Steps," in 2011 Cross Strait Quad-Regional Radio Sci. Wirel. Technol. Conf. IEEE, July 2011, pp. 498-501.

[20] S. Horst, D. E. Anagnostou, G. E. Ponchak, E. Tentzeris, and J. Papapolymerou, "Beam-Shaping of Planar Array Antennas Using Integrated Attenuators," in 2007 Proc. 57th Electron. Components Technol. Conf. IEEE, 2007, pp. 165-168.

[21] S. Yang and Z. Nie, "Time modulated planar arrays with square lattices and circular boundaries," Int. J. Numer. Model. Electron. Networks, Devices Fields, vol. 18, no. 6, pp. 469-480, November 2005.

[22] A. Bresler, "A new algorithm for calculating the current distributions of dolph-chebyshev arrays," IEEE Trans. Antennas Propag., vol. 28, no. 6, pp. 951-952, November 1980.

[23] D. Masotti, P. Francia, A. Costanzo, and V. Rizzoli, "Nonlinear/electromagnetic approach for time-modulated array simulation," in 2014 IEEE Radio and Wireless Symposium (RWS). IEEE, January 2014, pp. $118-120$.

[24] Y. Tong and A. Tennant, "A Two-Channel Time Modulated Linear Array With Adaptive Beamforming," IEEE Trans. Antennas Propag., vol. 60 , no. 1, pp. 141-147, January 2012.

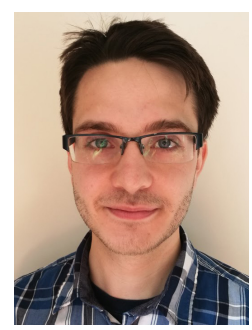

Ben Clark was born in Enfield, U.K., in 1991. He received the M.Eng degree in electronic and electrical engineering from Loughborough University, Loughborough, U.K., in 2014 and is currently a $\mathrm{PhD}$ candidate studying the uses of time-modulated arrays. His other research interests include sensor integration and signal processing.

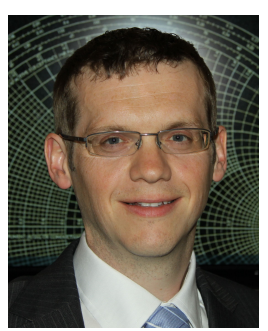

James A. Flint (M'96-SM'07) was born in Holbrook, Derbyshire, U.K., in 1973. He received the M.Eng. and Ph.D. degrees in electronic and electrical engineering from Loughborough University, Loughborough, U.K., in 1996 and 2000, respectively. He worked in the automotive industry as a Project Engineer and later returned to Loughborough when he was appointed Lecturer in Wireless Systems Engineering in 2001. He was promoted to Senior Lecturer in 2006 and Reader in 2017. His research interests span fault-tolerant signal processing, novel acoustic and electromagnetic transducers, metamaterials and electromagnetic compatibility. Dr. Flint is a Chartered Engineer in the U.K., a Fellow of the Institution of Engineering and Technology, and has acted as a consultant to numerous high profile companies and government agencies. 